\title{
AIP
}

\section{Explicit all-atom modeling of realistically sized ligand-capped nanocrystals}

Ananth P. Kaushik and Paulette Clancy

Citation: J. Chem. Phys. 136, 114702 (2012); doi: 10.1063/1.3689973

View online: http://dx.doi.org/10.1063/1.3689973

View Table of Contents: http://jcp.aip.org/resource/1/JCPSA6/v136/i11

Published by the AIP Publishing LLC.

Additional information on J. Chem. Phys.

Journal Homepage: http://jcp.aip.org/

Journal Information: http://jcp.aip.org/about/about_the_journal

Top downloads: http://jcp.aip.org/features/most_downloaded

Information for Authors: http://jcp.aip.org/authors

\section{ADVERTISEMENT}

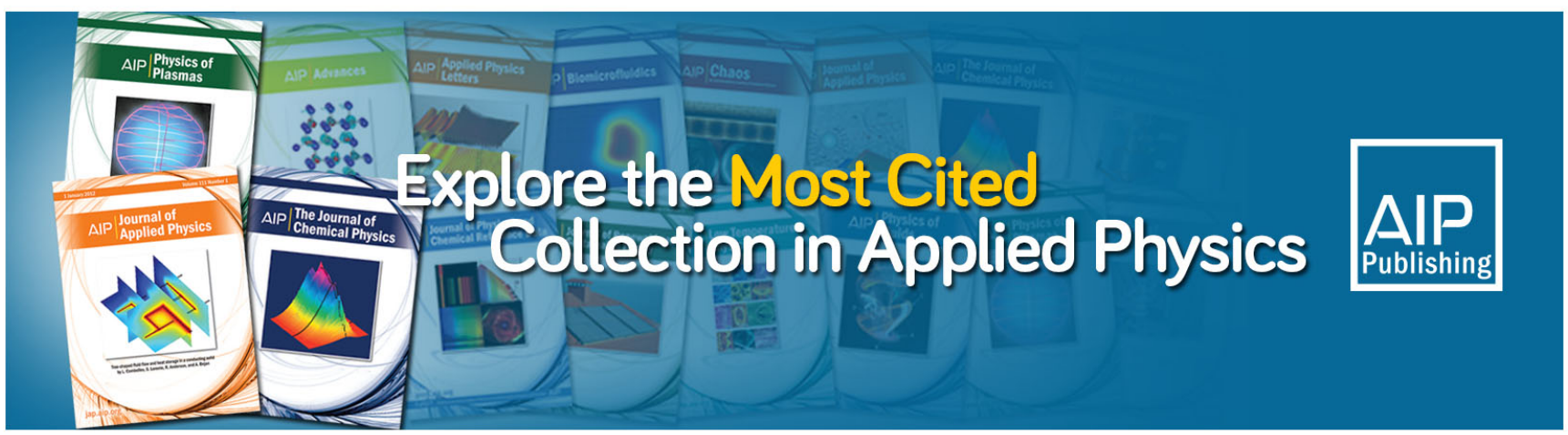




\title{
Explicit all-atom modeling of realistically sized ligand-capped nanocrystals
}

\author{
Ananth P. Kaushik and Paulette Clancy a) \\ School of Chemical and Biomolecular Engineering, Cornell University, Ithaca, New York 14853, USA
}

(Received 25 October 2011; accepted 30 January 2012; published online 15 March 2012)

\begin{abstract}
We present a study of an explicit all-atom representation of nanocrystals of experimentally relevant sizes (up to $6 \mathrm{~nm}$ ), "capped" with alkyl chain ligands, in vacuum. We employ all-atom molecular dynamics simulation methods in concert with a well-tested intermolecular potential model, MM3 (molecular mechanics 3), for the studies presented here. These studies include determining the preferred conformation of an isolated single nanocrystal (NC), pairs of isolated $\mathrm{NCs}$, and (presaging studies of superlattice arrays) unit cells of NC superlattices. We observe that very small NCs $(3 \mathrm{~nm})$ behave differently in a superlattice as compared to larger NCs (6 $\mathrm{nm}$ and above) due to the conformations adopted by the capping ligands on the NC surface. Short ligands adopt a uniform distribution of orientational preferences, including some that lie against the face of the nanocrystal. In contrast, longer ligands prefer to interdigitate. We also study the effect of changing ligand length and ligand coverage on the NCs on the preferred ligand configurations. Since explicit allatom modeling constrains the maximum system size that can be studied, we discuss issues related to coarse-graining the representation of the ligands, including a comparison of two commonly used coarse-grained models. We find that care has to be exercised in the choice of coarse-grained model. The data provided by these realistically sized ligand-capped NCs, determined using explicit all-atom models, should serve as a reference standard for future models of coarse-graining ligands using united atom models, especially for self-assembly processes. () 2012 American Institute of Physics. [http://dx.doi.org/10.1063/1.3689973]
\end{abstract}

\section{INTRODUCTION}

The nanoscale dimensions of "quantum dots," also known as nanocrystals (NCs), allow quantum confinement of electrons and holes, leading to the remarkable observation that the properties of quantum dots of the same material are size-dependent. Considerable research has been undertaken to try to create monodisperse arrays of nanocrystals in controlled geometries, not only to exploit their unusual or unique electronic properties, but also to fabricate practical devices for applications such as solar cells and sensors. ${ }^{1-5}$ The main "bottom up" synthesis method used to achieve such monodispersed NCs involves, first, "capping" the nanocrystals with surfactant ligands, often functionalized alkyl chains. These ligand coatings allow the nanocrystals to approach one another closely (self-assemble), without allowing neighboring nanocrystals to aggregate together, or sinter, since that destroys the confinement. We are ultimately interested in simulating "capped" chalcogenide nanocrystals, like CdSe or PbS, that are under intense current scrutiny as potential photovoltaic solar cell materials. ${ }^{6-9}$ For these systems, there are still some fundamental questions to ask of the self-assembly process, some of which this paper will attempt to answer.

In experiments, "capped" NCs assemble into superlattices of crystals in the 3-10 $\mathrm{nm}$ diameter range with facetted morphologies and near-perfect crystalline orientation. Difficulties in experimental control lead to the observation that the

a) Author to whom correspondence should be addressed. Electronic mail: Paulette.Clancy@cornell.edu. smaller the diameter of the nanocrystal, the larger the size dispersity of the array. Thus, in practice, experimentalists typically work in the $6-10 \mathrm{~nm}$ range. In contrast, as we shall show below, molecular simulation studies of such systems have almost exclusively been conducted for NCs whose diameters, $1-3 \mathrm{~nm},{ }^{10-13}$ are far smaller than typical experiments, presumably due to computer resource limitations. In addition, most of these studies have approximated the representation of the ligand chains, not as explicit all-atom models, but as "united atom" (UA)-like representations. ${ }^{10-14}$ For example, UA models represent a $-\mathrm{CH}_{2}$ group as a single entity, like a bead on a chain. As we shall show, any UA approximation has to be made judiciously for this critically important task. Explicit all-atom simulations of capped nanocrystalline arrays of realistically sized particles remain essentially intractable, except to those with access to petascale or highly parallelized computational resources.

Despite decades of experimental research to study capped NCs, the relative extent of the roles played by ligands and nanocrystals in the assembly process remains unclear, especially as the processing parameters are altered (e.g., temperature, choice of solvent, aging, and exposure to air). Indeed, even the nature of the binding between the ligands and the surface of the nanocrystal, and the density dependence of the ligands on different facets of the nanocrystal, is unknown. Further, there are few experimental probes that can truly answer these molecular-scale questions since they describe events at buried interfaces that are difficult to image or probe. This provides considerable motivation to employ molecular simulation approaches to shed some light on these complex 
questions, even though the scale of these systems in terms of length and time scales also represents cutting-edge challenges to simulation.

It is clear from previous molecular simulation studies ${ }^{10-14}$ that the ligands are not simply "spacers" between the nanoparticles in the superlattice to prevent sintering. They clearly play a defining role during the assembly process and in the resulting morphology of the superlattice. They also passivate surface states. Hence, the representation of the ligands is a critically important task. Indeed, impressively large-scale (at the time) molecular dynamics simulations by Luedtke and Landman ${ }^{10}$ in the 1990s described the interactions of sub-3-nm gold nanocrystals capped with thiol-terminated alkyl chains. Their results suggested that the gold-gold interaction contributed just $1 \%$ to the overall energy per particle of the system. This work was also notable for their prescient suggestion of the importance of the ratio of ligand length to nanoparticle diameter, $1 / \sigma$, to determine the preferred morphology (fcc vs. bcc, etc.), although they were unable to confirm this in their simulations given resource constraints. This is an issue we will take up here. On the other hand, they make a more disputable point of the tendency of ligands to "bundle" together at low temperatures. Badia et $a{ }^{15}$ claim to observe bundling for gold nanoparticles. Similar observations of bundling were made by Lane et al. ${ }^{16}$ when modeling spherical nanoparticles capped by alkanethiol ligands in water, decane, and in vacuum. More recent work by Schapotschnikow and Vlugt, ${ }^{11}$ also on sub-3-nm gold nanoparticles, was the first to include the effect of the role of the solvent to influence the adsorption of ligands during the assembly process, and the effect of the curvature of the gold surface on phase behavior. Lane et al. ${ }^{16}$ and Yang et al. ${ }^{17,18}$ have also studied solvent effects on alkane ligands attached to nanoparticles in different solvents, such as water and decane. We shall address solvent effects in a subsequent paper. Here, we consider only assembly in vacuo, which is the usual starting point for such simulations.

We will show in this paper that reports of bundling, i.e., ligands grouping together in distinct orientations on the surface of facetted nanocrystals, can be an artifactual result of the choice of the potential model used. We will compare three different potential models - one explicit and two united atom models to simulate our nanocrystal systems. One of the UA models used is the Jorgensen united atom model $^{19}$ that has been used by Luedtke and Landman ${ }^{10} \mathrm{We}$ will show that this model produces bundled conformations of ligands not produced by the explicit all-atom molecular mechanics 3 (MM3) model. Several papers have reported that the Jorgensen model overemphasizes attractive ligandligand interactions. ${ }^{20,25,26,28}$ We will hypothesize that the phenomenon may be the result of a combination of overattractive van der Waals' interactions and stiff dihedral interactions within the ligand molecules.

Computational efficiency has made commonplace the coarse-graining of (say) $-\mathrm{CH}_{2}$ groups into "united atoms" or "beads," thereby reducing the overall number of interacting entities in the system. ${ }^{10-14}$ One of the most important considerations when using the UA model is the quality of the interaction parameters chosen. The model should be able to reproduce, at least qualitatively, the trajectories of the system that were produced using the explicit all-atom method used before. Klein et al. reinforce this need and point to potential problems in their review article: ${ }^{14}$ "Outside of the polymer community, however, Scott points out in a recent review that existing CG [coarse-grained] models 'suffer from a lack of connection to atomistic interactions, which must ultimately be responsible for phase separation and domain formation.",27

One of the earliest, and still perhaps the most widely used, UA models was proposed by Jorgensen et al. in $1984^{19}$ which has been widely used to simulate various systems and has shown good results for liquid or liquid-like systems. However, as early as 1990 , Toxvaerd's studies on alkanes ${ }^{20}$ showed the tendency of the Jorgensen model to produce overly attractive interactions among alkyl chain molecules. He posited that the Jorgensen UA model is unable to describe the van der Waals interactions between the carbon atoms on the backbone of the alkyl chain and the adjacent hydrogen atoms, which are necessary to accurately describe the flexibility of the alkyl chain. He suggested using an "anisotropic" UA model, but this model is rather complicated to implement. Siepmann et $a .^{21}$ used configurational-bias Monte Carlo (MC) in the Gibbs ensemble to calculate vapor-liquid coexistence curves of $n$-alkanes containing 5-48 carbons. They found that the optimized potentials for liquid simulations (OPLS) force field overestimated the critical temperatures of the $n$-alkanes and proposed a new force field (Siepmann Karaborni and Smit (SKS)). This force field gave improved results for mediumto long- chain $n$-alkanes, but overestimated the critical temperatures of shorter alkanes. In 1998, they proposed a new "transferable potentials for phase equilibria" (TraPPE) force field, ${ }^{22}$ which proved to be superior to both OPLS (Ref. 29) and SKS (Ref. 23) in predicting phase equilibria; TraPPE is now widely used. The same year, Nath et al. introduced the NERD force field, ${ }^{23,24}$ which provided good agreement with experimental phase equilibria data not only for pure alkanes and alkenes, but also for their binary and ternary mixtures. Studies of a quite different system, polymers, in 2010 by $\mathrm{Li}$ et al., ${ }^{25}$ compared the conformation of polyethylene chains using an explicit Dreiding model ${ }^{28}$ with the Jorgensen et al.'s OPLS UA model. They observed that Jorgensen UA-modeled polyethylene chains adopted a lamellar conformation which was inflexible, while the same chain modeled with the allatom explicit Dreiding model was much more flexible and adopted more random conformations. They suggested a possible correction that involved increasing the $\sigma$ parameter of the van der Waals interaction for the carbon backbone to relieve some of the inflexibility of the alkyl chain. In contrast to Li et al.'s implication of intermolecular forces being at fault, Paul et al. ${ }^{26}$ looked instead at the intramolecular forces and offered an alternative approach to a united atom representation. In Sec. V, we will use Jorgensen et al.'s, Li et al.'s, and Paul et al.'s UA models and provide a comparison between the coarse-grained models that may be used to represent a system of alkyl ligand-capped nanocrystals.

In 2004, Kumar and co-workers produced an ambitious multiscale modeling study of the assembly of "capped" Co nanoparticles, starting from a simulation volume filled with Co atoms and ligand atoms. ${ }^{30}$ The study began with 
$a b$ initio calculations to provide an accurate starting point for the interactions between Co atoms and ligands and solvent. The next step involved a lattice-based MC simulation of NC assembly from Co atoms and surfactant ligands; this showed a preference to form 5-nm clusters and allowed the computation of an effective potential energy function between ligands. Finally, this second system of nanoparticles is coarse-grained such that each nanoparticle is reduced to a single point "particle" that interacts with other point "particles" through the effective potential obtained in step 2. Such coarse-graining facilitated the simulation of hundreds of nanoparticles (rather than hundreds of Co atoms in step 2). This elegant study, like all kinetic Monte Carlo methods, also has some limitations. For instance, the drawback of using an "on-lattice" approach and effective potential constraints means that subtler interactions could be lost. There are also issues regarding the modeling of the solvent-surfactant interactions and hence estimation of entropy effects. The most direct way to know how good a lattice-based approach will be in this situation is to explicitly model every atom in the capping ligands, the core nanoparticles, and the surrounding solvent. Unfortunately, for nanoparticles sizes that experimentalists can produce with a tight size distribution (5-10 nm diameter), such an explicit treatment is prohibitively expensive. An off-lattice kinetic Monte Carlo (KMC) approach could loosen these constraints, but currently available codes are also too expensive for such an undertaking. This remains a viable option for the future with a suitably constructed code.

\section{CONFIGURATION OF THE SYSTEM}

Our long-term interest lies in representing the properties of lead chalcogenide nanocrystalline arrays of varying diameters, passivated by long-chain organic ligands. The equilibrium structure for $\mathrm{PbSe} \mathrm{NCs}$ is a fcc lattice, which consists of two interpenetrating fcc lattices made of $\mathrm{Pb}$ and $\mathrm{Se}$ in the rock salt $(\mathrm{NaCl})$ structure. The lattice constant of the PbSe $\mathrm{NC}$ is $6.12 \mathrm{~nm}$. Following Luedtke and Landman's result that the ligands dominate $\mathrm{NC}-\mathrm{NC}$ interactions, and as a means to decouple ligand-ligand interactions from $\mathrm{NC}-\mathrm{NC}$ interactions, we focus in this study mainly on the ligand-ligand interactions, and the conformations adopted by the ligands on the $\mathrm{NC}$ surface. We will confirm the Landman result that core-core (NC-NC) interactions will be weak for the length of chains typically used in experimental self-assembly of chalcogenide systems (typically 12 carbon backbone atoms in length).

We shall identify a number of key parameters that govern the conformations of the ligands as well the interaction between ligands on the same NC and interaction between ligands on different NCs. In the experimental setup, NCs are grown in solution containing organic ligands as precursors. The ligands passivate the NC surface as the NC grows in solution. The size and shape of the NC is governed by the temperature of the solution and by the concentration of the precursors. Nanocrystals with very small diameters $(<3 \mathrm{~nm})$ are more or less octahedral in shape. In this study, we have approximated the shape of such small NCs as being perfect octahedra (Figure 1(b)) and hence exhibiting only $\{111\}$ facets, which are known to be purely $\mathrm{Pb}$-terminated. Larger NCs with
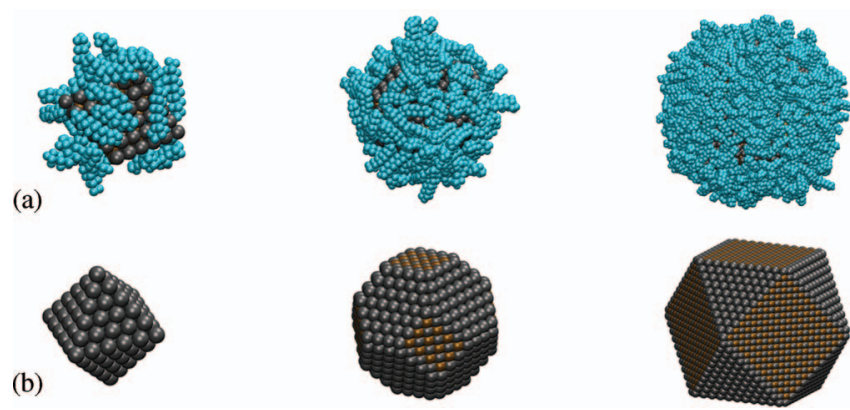

(b)
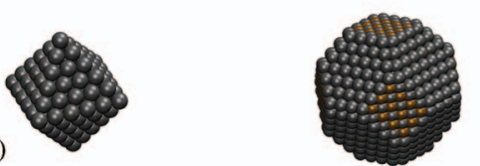

FIG. 1. (a) MD snapshots of the conformations of thermalized ligand-capped nanocrystals at $300 \mathrm{~K}$. From left to right: $3 \mathrm{~nm}, 4 \mathrm{~nm}$, and $6 \mathrm{~nm}$ diameter particles. The ligands are C12 ligands about $13 \AA$ long; ligand coverage is 3.3 ligands $/ \mathrm{nm}^{2}$. (b) The underlying shapes of the cores corresponding to each of the ligand-capped nanocrystals in Fig. 1(a).

diameters in the $4-7 \mathrm{~nm}$ range have both $\{100\}$ and $\{111\}$ facets. There is some evidence that there may also be $\{110\}$ surfaces around the corners and edges. ${ }^{31}$ Very large NCs (10 $\mathrm{nm}$ or higher) are almost cubic. ${ }^{32}$ In this study, we have assumed that the NCs of diameters of around $4 \mathrm{~nm}$ are truncated octahedra and larger NCs (diameters $6 \mathrm{~nm}$ ) have a cubeoctahedral shape with both $\{100\}$ as well as $\{111\}$ facets (see Figure $1(\mathrm{~b})$ ). The $\{100\}$ facets are made of alternating $\mathrm{Pb}$ and Se atoms, whereas the $\{111\}$ facets are Pb-terminated. This was chosen because there is experimental evidence that the oleic acid ligands typically used to passivate the NC surface have a greater preference for $\mathrm{Pb}$ atoms, thereby promoting the formation of $\mathrm{Pb}$-terminated $\{111\}$ facets. ${ }^{33}$

The organic ligands chosen for this study were aliphatic chains made of 12 carbon atoms and associated hydrogen atoms ("C12" ligands), since these are close in length to the oleic acid ligands often used by experimental studies of chalcogenide nanocrystals. We have also studied the effect of varying ligand length, in which case, ligands were used with chain lengths from $4 \mathrm{C}$ backbone atoms (a "C4" ligand) to ones with $18 \mathrm{C}$ backbone atoms (a "C18" ligand). The parameters for all the interactions between the ligands are described in Sec. III. The ligand molecules were created using the MOLDEN software package ${ }^{34}$ and an energy minimization of the initial guessed structure was performed using a standard minimization algorithm - the limited memory L-BFGS minimization using a modified version of the algorithm of Nocedal which is a part of the TINKER software package. ${ }^{35}$ The structures of other shorter and longer chain ligands were obtained in a similar manner. The ligands used in experiments are generally acids like oleic acid (C12) or thiols. The head group of the ligands attach themselves onto the $\mathrm{Pb}$ atoms of the NCs. In our system, we have represented the attachment of the head group by a single $\mathrm{C}-\mathrm{Pb}$ bond. The ligands were grafted onto the surface of the NC at a given density, typically the close-packed density for that facet. We have performed simulations where ligands were grafted with the entire carboxylic acid head group and we did not find any significant differences in the final morphology of the ligands compared to the case if we used a simpler $\mathrm{C}-\mathrm{Pb}$ bond for the grafting of the ligands onto the NC surface. Hence, all simulations reported in this study used the $\mathrm{C}-\mathrm{Pb}$ bond (which is a part of the TINKER package) to link the ligand with the $\mathrm{Pb}$ atoms on 
the NC surface. In essence then, we have a corona of ligands attached to lattice sites appropriate to the shape of a PbSe NC, but one in which the core is hollow, devoid of particles.

\section{INTERMOLECULAR POTENTIAL MODELS}

The choice of intermolecular potential model is a critical part of any molecular simulation. The inter- and intramolecular potential models and associated parameters determine the trajectory of the system and hence the morphology and conformations of the molecules in the simulation. As we shall describe in Sec. VI, the manner in which the coarsegraining is performed can have a profound effect on the different conformations adopted by the system. Hence, it is important that the system behaves in a reasonably consistent manner irrespective of the degree of coarse-graining or the choice of the potential model used. There exist few studies in literature that have been performed on nanocrystal systems with explicit all atom models ${ }^{16-18}$ and most studies have employed coarse-grained models. Thus, there is a lack of a "gold standard" from an explicit all atom simulation reference point to test the effectiveness of coarse-grained models. In this paper, we redress this situation and show that this was necessary to highlight some misleading structural properties that can be determined if the coarse-graining is not performed carefully.

We chose to use the non-reactive semi-empirical allatom explicit molecular mechanics 3 (MM3) potential, developed by Allinger et al., ${ }^{36}$ to model all the ligand-ligand interactions. Core-core interactions were not considered in these simulations (as discussed above in reference to Landman). The MM3 potential is an explicit all-atom potential similar to other explicit potentials such as OPLS-AA, Dreiding, CHARMM, etc. ${ }^{27,28,37}$ The MM3 potential has been shown, by us and others, ${ }^{38-43}$ to describe hydrocarbons quite accurately, ${ }^{36}$ in many cases, in good agreement with ab initio calculations. MM3 incorporates stretching, bending, and torsional energies, as well as the van der Waals interaction energies based on phenomenologically determined parameters. The total energy may be represented as follows:

$$
E=E_{b}+E_{a}+E_{t o r}+E_{a a}+E_{s b}+E_{s t o r}+E_{v d w}
$$

with

$$
\begin{gathered}
E_{b}=\frac{k}{2}\left(r-r_{0}\right)^{2}+\frac{k^{\prime}}{3}\left(r-r_{0}\right)^{3}+\frac{k^{\prime \prime}}{4}\left(r-r_{0}\right)^{4}, \\
E_{a}=\frac{k}{2}\left(\theta-\theta_{0}\right)^{2}+\frac{k^{\prime}}{3}\left(\theta-\theta_{0}\right)^{3}+\frac{k^{\prime \prime}}{4}\left(\theta-\theta_{0}\right)^{4} \\
+\frac{k^{\prime \prime \prime}}{5}\left(\theta-\theta_{0}\right)^{5}+\frac{k^{\prime \prime \prime \prime}}{6}\left(\theta-\theta_{0}\right)^{6} \\
E_{t o r}=\frac{k}{2}(1+\cos (\varphi))+\frac{k^{\prime}}{2}(1-\cos (2 \varphi)) \\
+\frac{k^{\prime \prime}}{2}(1+\cos (3 \varphi)), \\
E_{a a}=k\left(\theta-\theta_{0}\right)\left(\theta^{\prime}-\theta_{0}^{\prime}\right), \\
E_{s b}=k\left(\theta-\theta_{0}\right)\left(r_{1}-r_{10}+r_{2}-r_{20}\right),
\end{gathered}
$$

$$
\begin{gathered}
E_{\text {stor }}=\frac{k}{2}\left(r-r_{0}\right)(1+\cos (3 \varphi)), \\
E_{v d w}=A \exp \left[-\frac{r}{\rho}\right]+\frac{C}{r^{6}},
\end{gathered}
$$

where $E_{b}$ is the bond-stretch, $E_{a}$ is the angle-bend, $E_{t o r}$ is the torsion, $E_{a a}$ is the bend-bend potential, $E_{s b}$ is the stretch-bend potential, and $E_{\text {stor }}$ is the stretch-torsion potential. Here, $r$ is the distance between the two atoms linked through a bond, $\theta$ is the angle between three atoms, and $\varphi$ is the dihedral angle defined by four atoms. $\theta^{\prime}$ is the angle between three other atoms which are interacting with the first triad of atoms. $r_{1}$ and $r_{2}$ are the distances between the vertex atom and the two other atoms of a triad of atoms defined by an angle. The subscript 0 refers to equilibrium values. The potential does not involve electrostatic interactions. The intermolecular van der Waals interactions take the form of a Buckingham potential, modified with tapering polynomials so that the energy smoothly decreases to zero at a cutoff distance of $10 \AA . . k, k^{\prime}$, $k^{\prime \prime}, k^{\prime \prime \prime}, k^{\prime \prime \prime \prime}, A, \rho$, and $C$ are all constants. The values of these constants are given in the original MM3 paper by Allinger et $a .^{36}$ and were used unchanged in this paper.

We have used the MM3 model extensively to study the energetics and structural characteristics of an array of small organic semi-conducting molecules including the acenes, rubrene, diindenoperylene (DIP), sexiphenyl, and $\mathrm{C}_{60}{ }^{41,42}$ and have confidence in its ability to model small molecule organic systems. Our most recent study involved an extensive study of self-assembled monolayers ${ }^{43}$ that involved the simulation of long-chain organic molecules very similar to the ligands attached to the NCs considered here.

All-atom explicit models, such as MM3, are computationally very expensive. For example, a system of 50000 atoms simulated for a $100 \mathrm{ps}$ can take almost $24 \mathrm{~h}$ to complete when run on a single processor of a Dell 410 system with a $2.93 \mathrm{GHz}$ Xeon core. Given this expense, the simulations were limited to consideration of only one or two fully capped NCs in a given simulation cell. Thus, in order to simulate larger arrays of NCs, e.g., superlattices, it is necessary, for all practical purposes, to appropriately coarse-grain the system by (say) treating groups of $\mathrm{CH}_{2}$ units in the ligands as a "united atom." We shall describe in Sec. VI how this coarse-graining was achieved, as well as the potential models employed for such simulations.

\section{SIMULATION METHODOLOGY}

The time evolution of the system was followed using a molecular simulation approach employing a modified Beeman algorithm, which is currently a part of the freeware TINKER software package. ${ }^{35}$ As mentioned in Sec. II, optimized structures of the ligands were obtained from an energy minimization of an initial guess structures using a standard minimization algorithm, here, the limited memory L-BFGS minimization using a modified version of the algorithm of Nocedal. ${ }^{44}$ The core of the NC was approximated by a hollow shell where only the outermost layer of atoms was considered. The NC core is either fixed or treated as a rigid body, as required. As described before, three different NC core shapes 
were considered, depending on the size of the NC core (see Fig. 1). The 3-nm NCs are octahedral, the 4-nm NCs are truncated octahedra, and the 6-nm NCs are cube-octahedra. The ligands were attached to the $\mathrm{Pb}$ atoms of the core using a $\mathrm{Pb}-$ $\mathrm{C}$ bond. The grafting density of the ligands plays an important role in the interaction of the NCs, as will be described in Sec. V B 2. But, for most simulations, the grafting density was fixed. The ligands were grafted onto every other $\mathrm{Pb}$ atom on the surface, i.e., there is a free $\mathrm{Pb}$ atom between every two $\mathrm{Pb}$ atoms that are linked to a ligand. This leads to a greater density of ligands on the $\{111\}$ surfaces than the $\{100\}$ surfaces because of the presence of $\mathrm{Se}$ atoms in-between the $\mathrm{Pb}$ atoms on the $\{100\}$ surfaces. This choice of ligand grafting density and location was made from $a b$ initio studies of preferential ligand attachment sites. ${ }^{45}$

The first system we studied consisted of an isolated NC in vacuum; the main purpose for this rudimentary study was to determine whether ligand bundling occurs even for a single $\mathrm{NC}$, as Luedtke and Landman had suggested. The system was thermalized at $300 \mathrm{~K}$ using a Nosé-Hoover thermostat ${ }^{46-49}$ in the canonical (NVT) ensemble for a period of 200-300 ps using a time step of $1 \mathrm{fs}$. It was necessary to simulate (at least) 200 000-300 000 time steps in order to suppress significant fluctuations in temperature and energy and equilibrate the conformations adopted by the ligands. The simulation box used periodic boundaries in all three Cartesian directions, but the size of the box was made large enough to avoid the interaction of the NC with its images across the periodic boundaries. The core of the NC was held fixed during this time. Once the system was judged to be adequately thermalized, typically $1 \mathrm{~ns}$, the conformations of the ligands on the surface of the NC were used to determine radial and angular distributions of the ligands around the nanocrystal core. These observations are reported in Sec. V. This procedure was repeated for all three NC core shapes - the octahedron, truncated octahedron, and the cube-octahedron.

Systems involving two or more NCs were then studied; they were equilibrated in the same manner described above. The cores of the nanocrystals in these studies were frozen to investigate the ligand interactions alone. We identified several key parameters that affect the interaction energy and equilibrium distance; they include the shape and size of the NC, ligand length, and grafting density of the ligands. These observations are discussed in Sec. V. In order to study the true interactions between two nanocrystals, it is necessary to allow the nanocrystal cores to rotate freely. Such a study was performed where the centers of the cores of the nanocrystals were held fixed but the cores were allowed to rotate about the center. This study allowed us to calculate the potential of mean force (PMF), which is a very useful measure to quantify the interaction of the NCs. The mean force, $F_{\text {mean }}$, for two NCs separated by a distance $r$ is defined as ${ }^{50,51}$

$$
F_{\text {mean }}(r)=\frac{1}{2}\left\langle\left(\vec{F}_{2}-\vec{F}_{1}\right)\right\rangle \cdot \vec{r}
$$

where \langle\rangle denotes an ensemble average of configurations taken when the system is in equilibrium at a temperature $\mathrm{T}$ and $\vec{F}_{1}$ and $\vec{F}_{2}$ are the total forces acting on the $\mathrm{NC}_{1}$ and $\mathrm{NC}_{2}$, respectively, and $r$ is the vector connecting the centers of mass of the two NCs. The PMF can be obtained from the mean force as

$$
\varphi_{M F}(r)=\int_{r}^{\infty} F_{\text {mean }}(s) d s .
$$

A convenient way of evaluating the PMF is to attach a fictitious spring with a known spring constant between the NCs under considerations and then move them relative to one another at a constant velocity. This technique is called steered molecular dynamics (SMD), which exploits Jarzynski's equality that relates equilibrium free energy difference to irreversible work in a non-equilibrium system. ${ }^{52,53}$ The two NCs are initially separated to a distance at which there is no interaction between them. The NC cores are not frozen, but have the ability to translate as well as rotate about their centers of mass as a rigid body. The motion of NC cores was integrated using a constant-energy, NVE, microcanonical ensemble with rigid body dynamics. The ligands were maintained under "equilibrium" conditions at $300 \mathrm{~K}$; their motion was integrated using a constant-temperature, $N V T$, canonical ensemble. In this way, the entire system (NC core plus ligands) was maintained at thermal equilibrium over the entire course of displacement. The force of interaction between the two NCs was recorded as a function of time and the PMF was evaluated as the sum of the forces over the entire displacement of the NCs. The PMF was computed by averaging multiple trajectories over the same pulling path/reaction coordinate. The results are presented below for each of the NC shapes studied.

\section{RESULTS AND DISCUSSION}

\section{A. Isolated nanocrystal study}

We began by simulating a single, isolated nanocrystal in vacuum to establish "baseline" observations of the conformation of the ligands at equilibrium for different nanocrystal diameters ( $3 \mathrm{~nm}, 4 \mathrm{~nm}$, and $6 \mathrm{~nm}$ ). For this study, the core of the $\mathrm{NC}$ was fixed and the ligands were equilibrated at a temperature of $300 \mathrm{~K}$ for a period of $1 \mathrm{~ns}$. The energy and temperature of the system was observed to achieve a constant mean value with an acceptable standard deviation of less than $1 \%$ of the mean value. The system was deemed to be close to equilibrium at this point. The ligand conformations adopted by differently sized, isolated NCs are shown in Figure 1(a). The ligand conformations were observed for a range of temperatures from $150 \mathrm{~K}$ to $450 \mathrm{~K}$. No significant differences in ligand morphology were seen for different core shapes, sizes, and temperatures, except for the smallest NCs (described in more details in the next paragraph). The ligands, as expected, waved around much more actively at higher temperatures due to thermal excitation, whereas they were relatively sluggish at lower temperatures.

Ligand conformations on the larger NCs are markedly different from the $3 \mathrm{~nm} \mathrm{NC}$ (see Figure 1). Facets on the $3 \mathrm{~nm}$ NC are quite small and, consequently, can accommodate very few ligands. Indeed, the length of the ligand is comparable to the lateral dimensions of the facet itself. The ligands attempt to maximize their interaction with the NC facets and hence prefer to adopt a supine position with respect to 

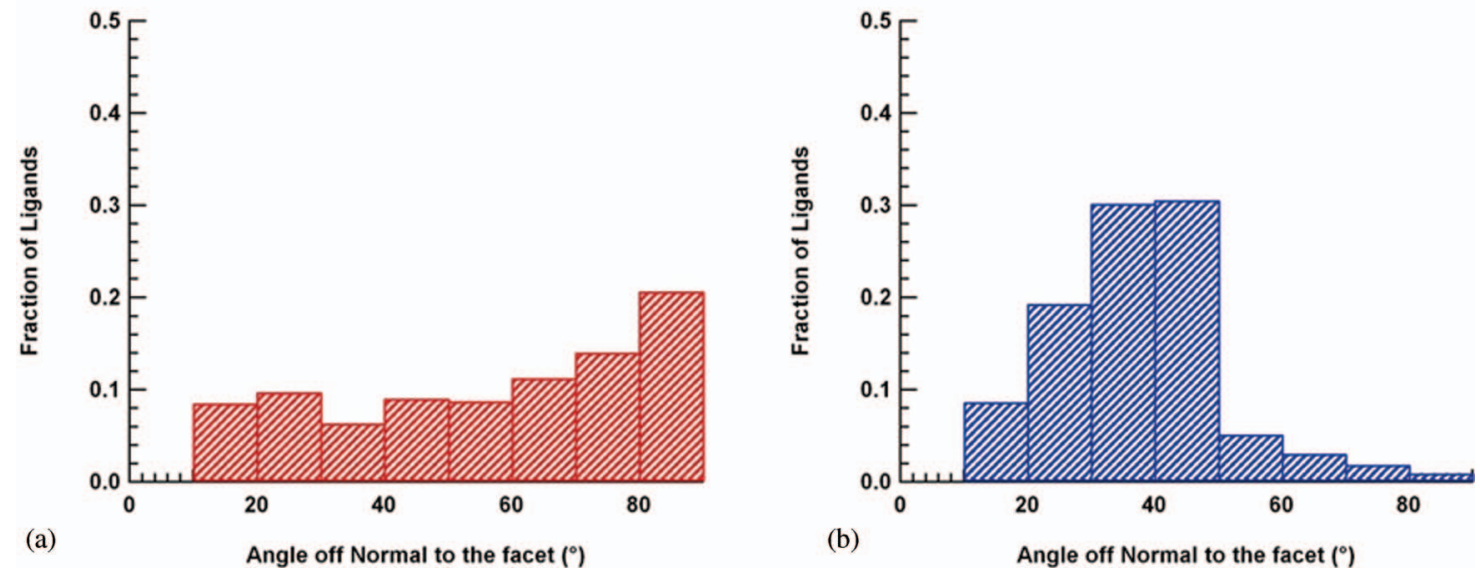

FIG. 2. Angular distribution of C12 ligands on the facets of (a) 3-nm and (b) 6-nm nanocrystals. The angle is measured as that made by the ligand with the normal to the facet of the NC to which it is attached. Ligands are about $13 \AA$ long. Coverage is 3.3 ligands $/ \mathrm{nm}^{2}$.

the facet. This can be seen in Fig. 1(a) where some of the ligands are wrapping around the NC core. Larger NCs have concomitantly larger facets and hence a larger number of ligands on the surface. This provides the ligands the opportunity to interact with each other, lessening ligand interactions with the core. The presence of many ligands on the surface also makes it essentially impossible for ligands to lie flat against the facet of the core due to steric hindrance from the other ligands. Thus, the ligands on the larger NCs adopt a more upright position as measured from the surface of the core. Since these conformations can be difficult to see from the MD snapshots, we compared graphs of the angular distribution of the ligands on the $3 \mathrm{~nm} \mathrm{NC}$ compared to the $6 \mathrm{~nm} \mathrm{NC}$, as shown in Fig. 2. The angles are measured as that made by the vector joining the ends of the ligands from the normal to the facet to which the ligands are attached. It can be clearly seen from the angular distribution profile that ligands wrap around the $\mathrm{NC}$ in the case of small NCs. The peaks in the $6 \mathrm{~nm} \mathrm{NC}$, correspond mainly to ligands standing upright on the corresponding $\{100\}$ and $\{111\}$ facets. No such peaks can be seen for the $3 \mathrm{~nm} \mathrm{NC}$; the distribution suggests that ligands adopt all possible angular conformations. This will lead to very different types of interactions between two or more NCs, as will be described in the Secs. V B and V C.

\section{B. Studies of pairs of NCs in a superlattice}

The interaction between two NCs that are not in the neighborhood of other NCs is different from the interaction between NCs in a superlattice. The interacting NCs in a "twonanocrystal" system, unfettered by the interaction of other $\mathrm{NCs}$, are free to sample many more conformations compared to NCs in a superlattice. In a superlattice, the NCs are rotationally and translationally constrained because of the presence of other nearby NCs. The ligands of the NCs drive the translational and rotational order of the NCs in the superlattice and, once the order has been established, the degrees of freedom of the NCs are severely constrained. Thus, in Secs. V B 1 and V B 2, we have examined the interaction between two NCs, not as an isolated system, but in a periodic superlat- tice by imposing periodic boundary conditions on the system. We have, however, examined an isolated "two-NC" system for comparison, which is described in Sec. V C.

Both bcc and fcc superlattice symmetries were chosen in our studies since these are the two most commonly adopted symmetries in experimental studies. Only one unit cell was simulated, using periodic boundary conditions to simulate infinite extent, thus maximizing computational efficiency. There are two NCs in the bcc unit cell, and four NCs in the fcc unit cell, as shown in Fig. 3. The NCs in the unit cells were initially far enough apart that the ligands on one $\mathrm{NC}$ did not interact with the ligands of the other NC. Thus, the distance between nearest neighbors in the unit cell was initially well beyond the cutoff used for the van der Waals interactions. The cores of the NCs were held rigid, while the ligands of the NCs were thermalized at a temperature of $300 \mathrm{~K}$ for $1 \mathrm{~ns}$. Once the equilibration period was over, the unit cell was compressed slowly at a rate of $1 \AA / n s$, while the system was constantly maintained in equilibrium at $300 \mathrm{~K}$. The compression process was thus as close to adiabatic (quasi-static) as possible. The compression of the system was interspersed by equilibration runs. The system was compressed for a period of $50 \mathrm{ps}$ and then equilibrated for the next $50 \mathrm{ps}$. This was done to ensure that the system was maintained in equilibrium, and fluctuations in energy and temperature were minimal. The ligands on the NCs were found to interdigitate very slowly as

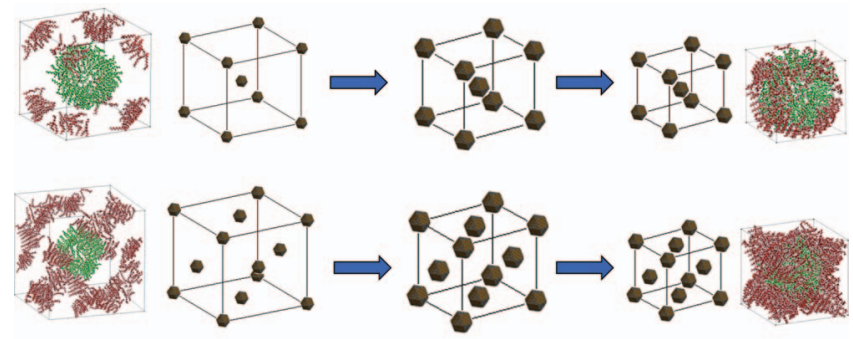

FIG. 3. Quasi-static compression of unit cells of a bcc lattice (top) and an fcc lattice (bottom). Initially, the brown ligands on the image cores are not in contact with the green ligands of the centrally located NC (LHS images). As the compression proceeds, the ligands eventually interdigitate, as shown in the MD snapshot images on the RHS. 


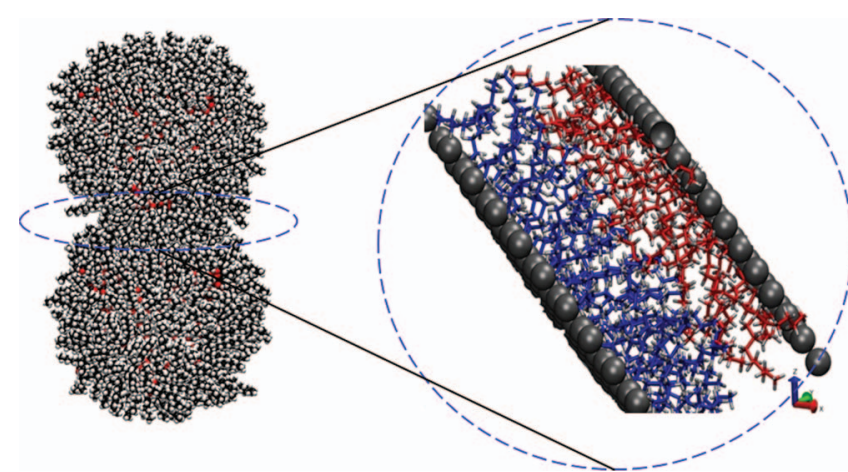

FIG. 4. Interdigitation of ligands between a pair of adjacent nanocrystal facets is shown on the expanded view (RHS image) of a two-NC cluster (LHS image). The ligands on different (identical) nanocrystals are shown as red and blue to facilitate observation of the extent of interdigitation.

the unit cell was compressed and the NCs were brought closer together as shown in Fig. 4. The interaction energy between the NCs displays a familiar Lennard-Jones-like curve with a long-range attractive portion, due to the favorable interaction between the ligands on the NCs, followed by a steeply repulsive portion due to the entropic repulsion between the interdigitated ligands. Compression of the unit cell was stopped once the repulsive portion of the curve was reached.

\section{Effect of ligand length}

In this section, we examined the effect of ligand length on the interaction between two NCs of size $3.1 \mathrm{~nm}$ in a periodic superlattice. The ligand grafting density was kept constant at 3.0 ligands $/ \mathrm{nm}^{2}$ which is in agreement with Ref. 45 . The length of the ligand was varied from $4 \mathrm{CH}_{2}$ backbone units to $18 \mathrm{CH}_{2}$ units. The parameters we examined were the separation distance between the centers of the NCs at the point of energy minimum $\left(\mathrm{r}_{\min }\right)$ and the value of the interaction energy at the energy minimum $(\varepsilon)$, as shown in Fig. 5. It is clear that both these curves are relatively linear for longer ligand lengths, specifically beyond $8 \mathrm{CH}_{2}$ units on the ligand. Thus, the nature of the inter-NC interaction is predictably similar for ligand lengths beyond $\mathrm{C} 8$, the difference arising merely from the addition of further $-\mathrm{CH}_{2}$ units. Similarly, the value of $\mathrm{r}_{\min }$ increases with ligand length because longer ligands cannot be compressed by the same amount as shorter ones. The interaction energy increases because there are more interaction sites on the ligand chain as the ligand length increases. The extent of interdigitation is generally minimal: Only the outermost $-\mathrm{CH}_{2}$ units of the ligands interdigitate due to the considerable steric hindrance, essentially regardless of ligand length (beyond about $\mathrm{C} 8$ ). The results for shorter ligand lengths (below $8 \mathrm{CH}_{2}$ units) deviate from this linearity. Short ligands interdigitate almost completely, so that the entire backbone of the ligand is involved in the inter-ligand interactions. Interdigitation increases as ligand length is reduced below C8.

This largely linear increase in $r_{\text {min }}$ with ligand length was not seen by Schapotschnikow et al. ${ }^{11}$ in MD studies of sub-3-nm-capped nanocrystals. In their studies, the value of $r_{\text {min }}$ showed variations about a mean value for different ligand lengths, leading them to suppose that the minimum of the potential of mean force is essentially independent of ligand length. This was not seen in our studies and we found that the longer the ligands, the farther out the potential minimum is in absolute distances. It is possible that, for very low grafting densities, or for the small spherical NCs used by Schapotschnikow et al., the potential minimum is essentially independent of ligand length because of the inherent flexibility of the ligands and the possibility of adopting conformations that avoid excess interaction. The introduction of sharp facets on the NCs could also account for the difference in behavior between our observations and those of Schapotschnikow et al., since our NCs are not spherical.

\section{Effect of grafting density of ligands}

We examined the effect of altering the grafting density of the ligands on the NC surface on the minimum interaction energy $(\varepsilon)$ (i.e., when the interaction energy of the ligands reaches a minimum with reducing inter nanocrystal separation) and the corresponding inter-NC separation distance $\left(r_{\min }\right)$. The NC diameters were 3, 4, and $6 \mathrm{~nm}$ for this study. In this simulation, the ligands were grafted at random points on the NC surface. This lack of order of the ligand attachment
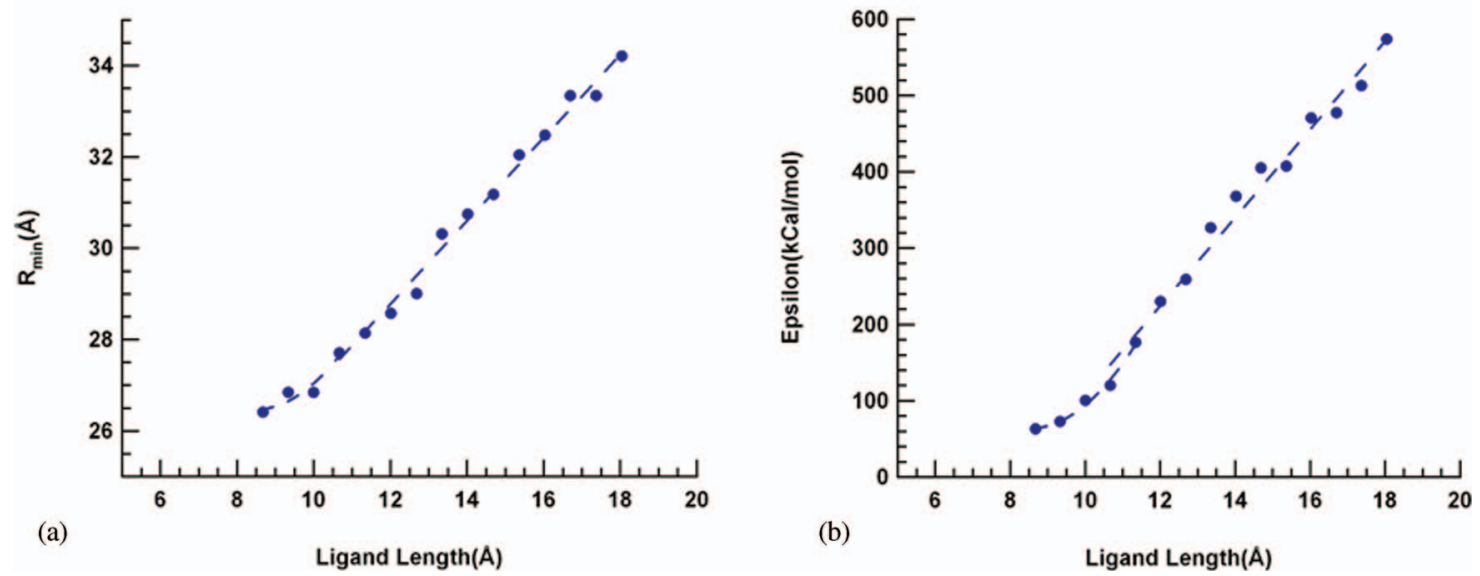

FIG. 5. Effect of ligand length on the values of the interaction energy, $\varepsilon$, and the separation distance, $\mathrm{r}_{\text {min }}$, corresponding to the potential minimum (PMF). (a) Separation distance and (b) interaction energy. Note the linearity of results for chains above about C8 in length. 

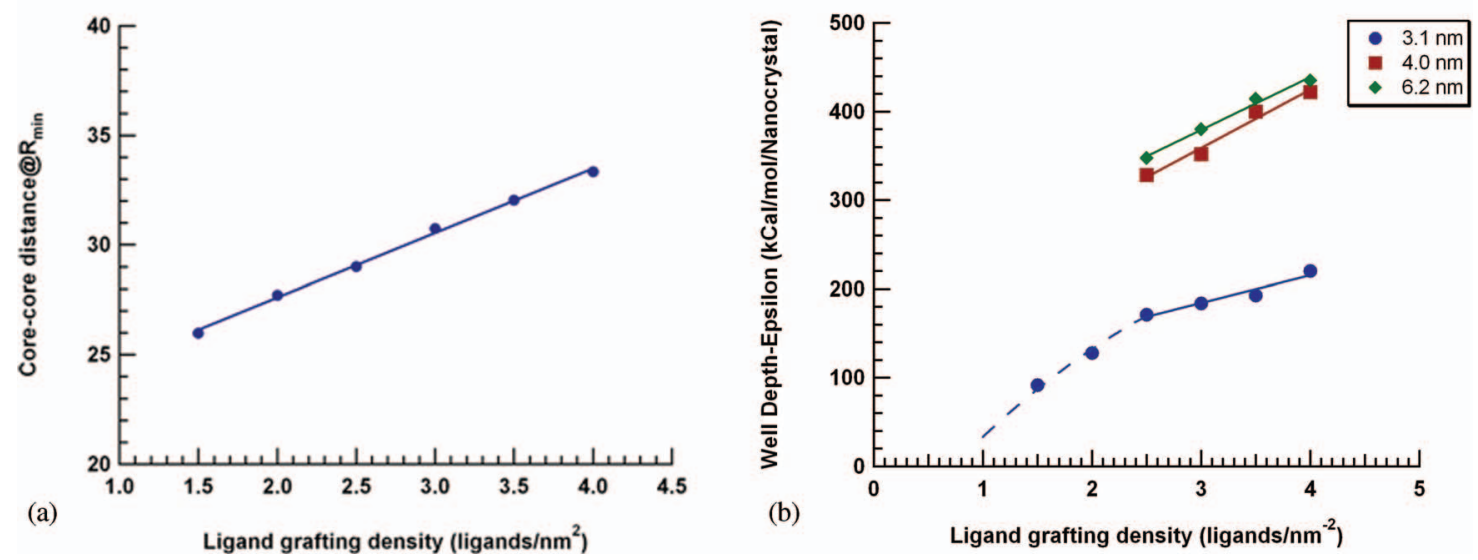

FIG. 6. Effect of ligand grafting density on (a) separation distance (for a $3.1 \mathrm{~nm} \mathrm{NC}$ ) and (b) interaction energy at the potential minimum.

on the nanocrystal core was adopted because experimentalists cannot readily observe the extent of any preference for ligand attachment on different facets. Hence, the assumption of a random pattern is a reasonable starting point.

The ligand length was kept constant at $12-\mathrm{CH}_{2}$ units, while the grafting density was varied from 1.5 ligands $/ \mathrm{nm}^{2}$ to 4.0 ligands $/ \mathrm{nm}^{2}$. This is essentially the entire accessible range up to a close-packed density. Plots of these key parameters for the ligand-ligand interaction energy $\left(\varepsilon\right.$ and $\left.r_{\min }\right)$ as a function of grafting density are shown in Fig. 6 . The value of $r_{\min }$ increases linearly with increasing grafting density. The "footprint" of the ligands on the NC facets increases in a linear fashion with grafting density. Thus, the number of ligands on a facet interacting with other ligands also increases linearly, preventing the NCs from approaching each other closely. The ligands adopt a more supine conformation on the facets at low density, whereas they stand more upright off the facets at high grafting density. The interdigitation of the ligands becomes more difficult with increasing grafting density, since the steric hindrance from the ligands increases the repulsive interactions. This leads to an increase in $r_{\min }$ with grafting density.

The minimum interaction energy, on the other hand, displays a more interesting behavior: It is linear for grafting densities from 2.5 to 4.0 ligands $/ \mathrm{nm}^{2}$. Below 2.5 ligands $/ \mathrm{nm}^{2}$, the minimum interaction energy, $\varepsilon$, decreases. The linear behavior arises, as for the effect of ligand length seen in Fig. 5, because the addition of more ligands onto the facet means that more $-\mathrm{CH}_{2}$ groups can interact, increasing the minimum interaction energy. For low grafting densities, ligand-ligand interactions on the $\mathrm{NC}$ facet become small, and ligand-core and core-core interactions become more important. Importantly, however, the dominant mechanism of ligand-ligand interactions at sufficiently low ligand densities (of up to around 2.5 ligands $/ \mathrm{nm}^{2}$ ) is no longer through interdigitation of the ligands. The lack of ligands allows some of them to adopt a more supine conformation with respect to the facet, and hence, the ligands on opposing $\mathrm{NC}$ facets interact with almost the entire backbone of the ligand chain. The ligands are sparse enough to allow a lot of compression of the ligand corona so that the minimum interaction energy $(\varepsilon)$ deviates from the linear profile seen for larger grafting densities. This has implica- tions for the stability of superlattices, since the stabilization is improved when the ligands interdigitate rather than when they are simply compressed. Thus, it is better if the grafting density of the ligands is reasonably large. On the other hand, very large grafting densities do not allow interdigitation at all which, once again, is detrimental to superlattice stability. These results suggest that some intermediate value for the density would lead to optimal interdigitation and packing.

\section{Potential of mean force for a two-nanocrystal system}

We used the PMF technique described in Sec. IV to evaluate the interaction between two isolated NCs as a function of the distance between them. The PMF was evaluated using the SMD feature of LAMMPS software package. ${ }^{54}$ The final PMF was the average of ten different simulation trajectories over the same pulling distance. The averaging ensures that unwanted oscillations and noise in the data are damped out. The nature of the PMFs of three different diameters of NCs was evaluated, as shown in Fig. 7. In order to calculate the PMF, it is important for the NCs in these simulations not to be rotationally confined. Accordingly, the NCs were allowed to translate as well as rotate about their centers of mass.

The PMFs show some very interesting features. For all three NC diameters, the PMF exhibits a minimum at a characteristic distance $\left(\mathrm{r}_{\min }\right)$ and then the curve rises sharply as the NCs are brought closer than this optimum distance. But, it may be observed that the curves for the NCs behave differently around the minimum: For the smallest NCs $(3 \mathrm{~nm}$ diameter), the curve is rather broad around the minimum and shallower than the other curves. In fact, the curves get deeper and narrower with increasing NC size. In other words, the interactions become "harder." The change in the shape of the potential well is due to the different mechanism of interaction in the case of the small NCs as compared to the larger NCs. The two dominant mechanisms are inter-(chain) backbone interactions versus inter-(chain) tip interdigitation. As described in Sec. V A, the small NC has very little surface area of $\{111\}$ facets and there are very few ligands on the facets. As a consequence, the ligands lie almost flat on the facets to maximize their interaction with the facets. 


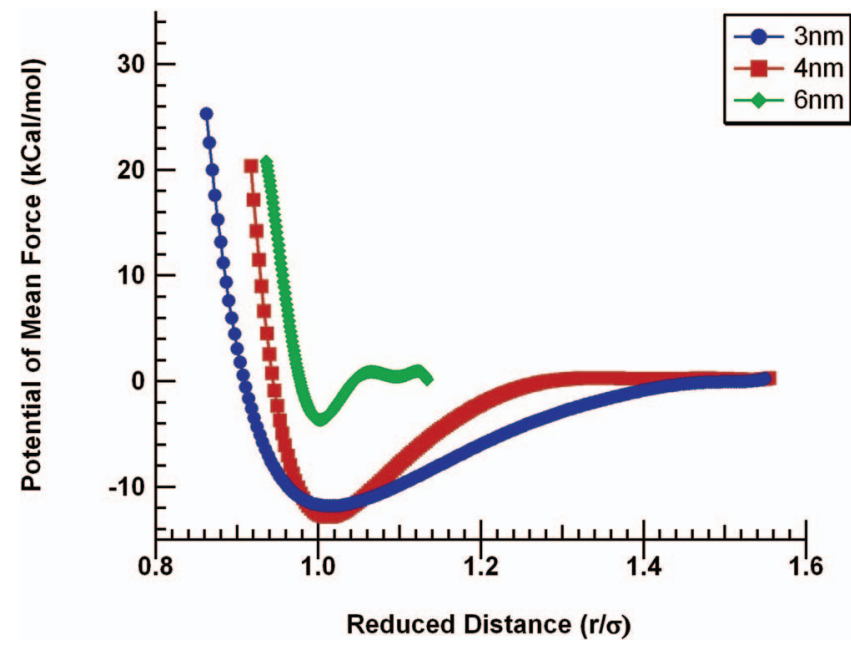

FIG. 7. Potential of mean force of ligand-capped nanocrystals for different nanocrystal diameters as a function of the reduced distance between the nanocrystals, given as the actual distance divided by the distance at energy minimum. The C12 ligands are about $13 \AA$ long; coverage is about 3.3 ligands $/ \mathrm{nm}^{2}$.

When the two NCs are brought closer together, the ligands on the respective NCs are able to interact with the full length/backbone of the ligand. As the interaction continues over the distance separating the facets of the two NCs, the ligands have the freedom to adopt different conformations to minimize the steric hindrance and maximize the interaction with ligands on the other NC. There is interplay between the interaction of the ligands and adoption of more favorable conformation of the ligands on the facets of the two NCs, creating the shallow broad potential energy minimum, which we believe is characteristic of largely supine ligands. This situation is depicted in Fig. 7. The repulsive part of the curve is not felt until the NC cores are quite close and at a point where the cores begin to interact as well. Thus, in the 3-nm-diameter $\mathrm{NC}$ case, both the ligands and the cores interact, though the cores are not close enough to contribute significantly to the total interaction. This is also the reason why the minimum in the PMF occurs at a much smaller distance of separation compared to the PMF curves of the larger NCs.

For larger $(\mathbf{6} \mathbf{~ n m}) \mathrm{NCs}$, the ligand-ligand interaction mechanism is somewhat different. The facets on these NCs are larger and can accommodate more ligands. This means that ligands on a facet interact with themselves quite strongly and do not need to maximize their interaction with the underlying core. Thus, the ligands tend to "stand up" and away from the surface of the facets of the core. They are also somewhat restricted in their freedom to adopt all of the possible conformations because of steric hindrance from other ligands on the facets. The interaction between the ligands on adjacent NCs arises mainly from interactions of the top few $\mathrm{CH}_{2}$ groups (the tips of the chains); the ligands are unable to "feel" the entire backbone of the ligand chain. The interplay described for $3 \mathrm{~nm}$ particles, i.e., the interaction of ligands versus the adoption of more favorable conformations by the ligands, is more constrained for larger $6 \mathrm{~nm}$ NCs since the ligands have very little conformational "space" to continually adapt to a more favorable conformation as the two NCs are brought closer to- gether. The PMF thus becomes sharper; the repulsive part of the curve begins almost immediately after the minimum is reached and is a lot steeper. Ligands on adjacent NCs interdigitate to maximize their interactions and minimize entropic repulsion, a mechanism not seen in the case of the $3 \mathrm{~nm} \mathrm{NCs}$. This can be seen in Fig. 7 for the 6-nm-diameter particles. The 4-nm NC showed characteristics intermediate between those of smaller (3-nm) and larger (6-nm) NCs. The strength of the interaction also depends on the number of ligands on the facets and, since the larger NCs have a larger facet area, they consequently also have a larger number of ligands, which leads to a stronger interaction at the potential minimum. The minimum in the potential occurs at a larger value of $r_{\text {min }}$ since the ligands start interacting strongly from a larger distance.

In larger arrays and superlattices of NCs, we shall show below that the ligands can interlock, providing mechanical stability to the superlattice. We believe that this is the reason for the greater ease of formation of stable NC superlattices with larger NCs than with, say, small 3-nm NCs.

\section{COARSE-GRAINING OF LIGANDS ON THE NANOCRYSTAL SURFACE}

The explicit all-atom model, used in all the preceding studies in this paper, provides detailed information about the nanocrystal interactions. Unfortunately, the number of interatomic interactions involved is very large, whether considering intermolecular or intramolecular terms. Thus, very quickly, it becomes infeasible to simulate larger systems of NCs.

One technique to overcome this limitation is the method of coarse-graining the representation of the ligands to a less atomically detailed description. A popular method of coarsegraining invokes the UA model, described in the Introduction of this paper. We examine two different UA models adopted from literature. The equations describing Jorgensen's UA model potential ${ }^{19}$ are given below. The parameters of this model are given in Table I of Ref. 59:

$$
\begin{gathered}
E_{\text {bond }}=K\left(r-r_{0}\right)^{2}, \\
E_{\text {angle }}=K^{\prime}\left(\theta-\theta_{0}\right)^{2}, \\
E_{\text {torsion }}=K_{1}(1+\cos (\varphi))+K_{2}(1-\cos (2 \varphi)) \\
+K_{3}(1+\cos (3 \varphi)), \\
E_{v d w l}=4 \varepsilon\left[\left(\frac{\sigma}{r}\right)^{12}-\left(\frac{\sigma}{r}\right)^{6}\right] .
\end{gathered}
$$

Here, $r$ is the bond distance between two bonded atoms, $\theta$ is the angle between three atoms, and $\varphi$ is the dihedral angle between four atoms. The subscript 0 refers to the equilibrium values of bond length and angle between the bonded atoms. $K, K^{\prime}, K_{1}, K_{2}$, and $K_{3}$ are constants. Equation (14) is the intermolecular interaction represented by a Lennard-Jones (126) potential; $\sigma, \varepsilon$, and $r$ represent the diameter, well depth, and distance between the two interacting atoms. Geometric mixing rules are used to define the cross interaction between 

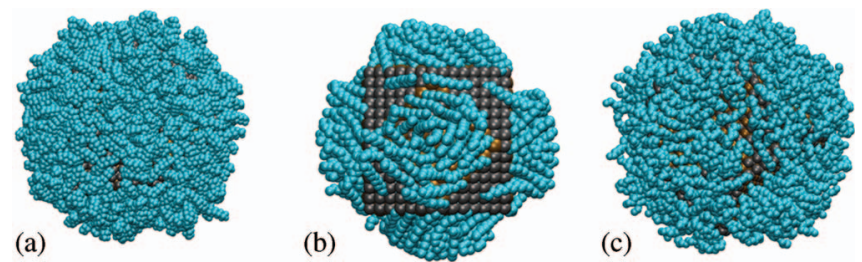

FIG. 8. Comparison of snapshots from MD simulations using (left to right, top panel) the results of the (a) explicit all-atom MM3 model (Ref. 36), with (b) united atom models due to Jorgensen et al. (Ref. 19) and (c) Paul et al. (Ref. 26). The C12 ligands are $13 \AA$ long; ligand coverage is 3.3 ligands $/ \mathrm{nm}^{2}$.

dissimilar interacting pairs of atoms. ${ }^{55}$ The cutoff distance used for the van der Waals interactions is $10 \AA$.

If the Jorgensen et al. UA model is used to describe our system of NCs, the final configuration of the ligands on an isolated nanocrystal after equilibration at $\mathrm{T}=300 \mathrm{~K}$ is shown in Fig. 8. For comparison, the same isolated nanocrystal, modeled using the explicit MM3 model is also shown. It can be seen clearly that ligands modeled with the Jorgensen UA model exhibiting "bundling" that is absent in the all-atom MM3 description. Ligands on each of the faces of the NC interact with each other very strongly and evince a preferential aggregation. Furthermore, the ligands showed very little dynamic motion at $300 \mathrm{~K}$, and preferred to stay at the equilibrium position at $300 \mathrm{~K}$, as shown in the figure. Bundling has also been observed by Landman ${ }^{10}$ and Glotzer ${ }^{56}$ using the Jorgensen model. In fact, Landman observed the bundling phenomenon even for isolated NCs (at $1 \mathrm{~K}$ and from 250 to $300 \mathrm{~K}$ ). Our experimental partners in the Hanrath group at Cornell have looked specifically for evidence of bundling through detailed analyses ${ }^{57}$ and their conclusion, like the allatom simulations presented here, is that there is insufficient experimental evidence to support the existence of bundling in these systems at room temperature.

Our results suggest that the parameters of the Jorgensen UA model may not estimate the temperature at which ligands disorder very well for the system under consideration here. The bundling phenomenon may occur for very long polymeric chains in a very poor solvent. Such ligand morphologies have been observed by Lane and Grest. ${ }^{16}$ But, for the system at hand, the Jorgensen model was unable to duplicate the ligand conformations of the explicit MM3 model. Since the so-called "melting temperature" of the ligands may be exaggerated by the Jorgensen model, we heated the isolated nanocrystal by running $N V T$ simulations at higher temperatures to equilibrate the system, followed by $N V E$ runs at the same higher temperatures. We saw the first hints of "un-bundling" of the ligands at a temperature of around $600 \mathrm{~K}$, well above the $300 \mathrm{~K}$ studies that are relevant for the study presented here (and at which experiments are conducted).

Our task then was thus to sort among several existing UA models that have been posited to pick one that would give similar ligand conformations as seen using the MM3 model which we believe to be representative of experiments. We started with Li et al.'s suggestion ${ }^{25}$ that an increase in the $\sigma$ parameter of the van der Waals interaction would solve the problem. Unfortunately, the bundling phenomenon persisted after equilibration as we varied $\sigma$ over what we assumed to be a reasonable range (from 3.5 to $4.2 \AA$ ). Refitting the van der Waals parameters to match the interaction energy of the equivalent interactions in the explicit MM3 model for simulations of an isolated $\mathrm{NC}$ in vacuum at $300 \mathrm{~K}$ did not remove the bundling effect. This led us to believe that the problem lay in the intramolecular parameters, namely, in the dihedral interactions. Further evidence implicating the overly strong dihedral interactions in the Jorgensen model is provided by observing that the OPLS-AA (an explicit all-atom Jorgensen model) shows the same sort of bundling as for the UA model. Thus, coarse-graining is not responsible for bundling, but the intramolecular interactions themselves (see the supplementary material). We therefore, used the UA model as defined by Paul et al. ${ }^{26}$ The equations describing their optimized UA potential are given below. Parameters for this model are given in Table II of Ref. 59:

$$
\begin{gathered}
E_{\text {bond }}=\frac{1}{2} k\left(r-r_{0}\right)^{2}, \\
E_{\text {angle }}=\frac{1}{2} k^{\prime}\left(\cos (\theta)-\cos \left(\theta_{0}\right)\right)^{2}, \\
E_{\text {torsion }}=\frac{1}{2} k_{1}(1-\cos (\varphi))+\frac{1}{2} k_{2}(1-\cos (2 \varphi)) \\
+\frac{1}{2} k_{3}(1-\cos (3 \varphi)), \\
E_{v d w l}=4 \varepsilon\left[\left(\frac{\sigma}{r}\right)^{12}-\left(\frac{\sigma}{r}\right)^{6}\right] .
\end{gathered}
$$

Here, $r$ is the bond distance between two bonded atoms, $\theta$ is the angle between three atoms, and $\varphi$ is the dihedral angle between four atoms. The subscript 0 refers to the equilibrium values of bond length and angle between the bonded atoms. $k$, $k^{\prime}, k_{1}, k_{2}$, and $k_{3}$ are constants. The last equation is the intermolecular interaction represented by a (12-6) Lennard-Jones potential; $\sigma, \varepsilon$, and $r$ represent atomic diameter, well depth, and distance between the two interacting atoms. Standard Lorentz-Berthelot mixing rules are used to define the cross interaction between dissimilar interacting pairs of atoms. ${ }^{55}$ The cutoff distance used for the van der Waals interactions is $10 \AA$.

The Paul et al. model, tested using the same conditions described above for an isolated $\mathrm{NC}$ at $300 \mathrm{~K}$, did not show any ligand bundling. Paul-modeled ligands adopted more random configurations and showed appropriate dynamic motion upon equilibration at $300 \mathrm{~K}$ similar to the MM3 simulations. Images of the ligand conformations for the MM3 model, the Jorgensen model, and the Paul et al. model are shown in Figure 8.

\section{CONCLUSIONS}

We have presented an explicit all-atom representation of nanocrystals, "capped" with alkyl chain ligands, of experimentally relevant sizes in vacuum. The studies included an isolated single NC, pairs of isolated NCs, and (presaging studies of superlattice arrays) unit cells of NC superlattices. The behavior of these ligand-capped NCs, determined by the 
explicit all-atom model, can serve as a reference standard for future coarse-graining of ligands using united atom models.

We have shown that several different parameters that control the conformations adopted by the ligands can lead to the same mechanism of interaction between the NCs. For instance, the shape of the attractive "bowl" of the PMF depends on the size (and coupled shape) of the NCs: Ligands on very small $(3 \mathrm{~nm}) \mathrm{NCs}$ do not interdigitate; they tend to wrap around the core and then compress. Ligands on larger NCs $(6 \mathrm{~nm})$ only interdigitate and then lock the NCs into place with very little compression. Ligands on intermediate-sized (4 nm) NCs exhibit some interdigitation and some ligand wrapping. A similar range of possible outcomes for the conformations adopted by dendrimers was observed in a paper by Li et al. ${ }^{58}$ They classified the conformation of ligands on the cores of their systems according to different hairstyles, ranging from smooth hairstyles (which they dubbed as "Valentino"), to asymmetric or "punk" hairstyles, to "Einstein" hair where the ligands stick out from the core. These classifications convey essentially the same picture as our wrapped/wrappedinterdigitated/interdigitated mechanisms for the 3, 4, and $6 \mathrm{~nm}$ diameter systems, respectively. These mechanistically driven results can also be obtained by varying the ligand density or the length of the ligands. Thus, by changing one or more parameters affecting the ligand conformations on the NC cores, we shed some light onto a path by which one could intelligently achieve the desired selfassembly of these NC systems. It also helps explain the inherent complexity of the behavior of real nanocrystal systems.

Coarse-graining is an excellent method of reducing computational complexity in order to save computational time. It provides the ability to model larger NC superlattices over longer time scales. All-atom modeling is essentially limited to systems not much bigger than the ones studied here. We found that the specific choice of the united atom potential model is crucial for the prediction of properties of the $\mathrm{NC}$ system. We experimented with two different UA models selected from literature. The Jorgensen UA model did not adequately capture the dynamics of these NC systems as described by the explicit MM3 model. We also found that the model introduced bundled ligand conformations which were not predicted by the MM3 model. We believe that this may be due to different intermolecular van der Waals force parameters, but mostly because of the difference in stiffness introduced by dihedral interactions in the ligands. We found that the Paul et al. UA model mimics the trajectories of the explicit MM3 model sufficiently well. Since reproducing the flexibility of ligands and the conformations of the ligands described by the explicit model is crucial to the study of NC systems, this model may be more suitable to model large NC systems such as the one we have described. At the very least, one must be circumspect, while transitioning from explicit models to coarse-grained models, in choosing one coarse grained model over another.

The obvious next steps are to move from small unit cell all-atom representations to superlattices and to explore the effect of having a solvent present. These simulations will be described in a subsequent paper.

\section{ACKNOWLEDGMENTS}

This publication was based on work supported by the Award No. KUS-C1-018-02, made by the King Abdullah University of Science and Technology (KAUST). The Hanrath research group at Cornell is thanked for access to their experimental data in advance of publication. Intel Corporation is thanked for the donation of computing resources crucial to the studies performed here.

${ }^{1}$ E. V. Shevchenko, D. V. Talapin, N. A. Kotov, S. O’Brien, and C. B. Murray, Nature (London) 439, 55 (2006).

${ }^{2}$ A. E. Saunders and B. A. Korgel, Chem. Phys. Chem. 6, 61 (2005).

${ }^{3}$ M. Grzelczak, J. Vermant, E. M. Furst, and L. M. Liz-Marzán, ACS Nano 4, 3591 (2010).

${ }^{4}$ J. Y. Ku, D. M. Aruguete, A. P. Alivisatos, and P. L. Geissler, J. Am. Chem. Soc. 133, 838 (2011).

${ }^{5}$ Y. Lin, A. Böker, J. He, K. Sill, H. Xiang, C. Abetz, X. Li, J. Wang, T. Emrick, S. Long, Q. Wang, A. Balazs, and T. P. Russell, Nature (London) 434, 55 (2005).

${ }^{6}$ S. Shrestha, Prog. Photovoltaics 19, 123 (2011).

${ }^{7}$ Z. Quan, L. Valentin-Bromberg, W. S. Loc, and J. Fang, Chem. Asian J. 6, 1126 (2011).

${ }^{8}$ H. W. Hillhouse and M. C. Beard, Curr. Opin. Colloid Interface Sci. 14, 245 (2009).

${ }^{9}$ J. H. Bang and P. V. Kamat, ACS Nano 3, 1467 (2009).

${ }^{10}$ W. D. Luedtke and U. Landman, J. Chem. Phys. 100, 13323 (1996).

${ }^{11}$ P. Schapotschnikow, R. Pool, and T. J. H. Vlugt, Nano Lett. 8, 2930 (2008).

${ }^{12}$ P. Schapotschnikow, M. A. van Huis, H. W. Zandbergen, D. Vanmaekelbergh, and T. J. H. Vlugt, Nano Lett. 10, 3966 (2010).

${ }^{13}$ P. Schapotschnikow and T. J. H. Vlugt, J. Phys. Chem. C 114, 2531 (2010).

${ }^{14}$ S. O. Nielsen, C. F. Lopez, G. Srinivas, and M. L. Klein, J. Phys.: Condens. Matter 16, R481 (2004).

${ }^{15}$ A. Badia, L. Cuccia, L. Demers, F. Morin, and R. B. Lennox, J. Am. Chem. Soc. 119, 2682 (1997).

${ }^{16}$ J. M. D. Lane and G. S. Grest, Phys. Rev. Lett. 104, 235501 (2010).

${ }^{17}$ A. Yang and C. Weng, J. Phys. Chem. C 114, 8697 (2010).

${ }^{18}$ A. Yang, C. Weng, and T. Chen, J. Chem. Phys. 135, 034101 (2011).

${ }^{19}$ W. L. Jorgensen, J. D. Madura, and C. J. Swenson, J. Am. Chem. Soc. 106, 6638 (1984).

${ }^{20}$ S. Toxvaerd, J. Chem. Phys. 93, 4290 (1990).

${ }^{21}$ J. I. Siepmann, S. Karaborni, and B. Smit, Nature (London) 365, 330 (1993).

${ }^{22}$ M. G. Martin and J. I. Siepmann, J. Phys. Chem. B 102(14), 2569 (1998).

${ }^{23}$ S. K. Nath, F. A. Escobedo, and J. J. de Pablo, J. Chem. Phys. 108, 9905 (1998).

${ }^{24}$ S. K. Nath, B. J. Banaszak, and J. J. de Pablo, J. Chem. Phys. 114, 3612 (2001).

${ }^{25}$ C. Li, P. Choi, and P. R. Sundararajan, Polymer 51, 2803 (2010).

${ }^{26}$ W. Paul, Do Y. Yoon, and G. D. Smith, J. Chem. Phys. 103, 1702 (1995).

${ }^{27}$ H. L. Scott, Curr. Opin. Struct. Biol. 12, 495 (2002).

${ }^{28}$ S. L. Mayo, B. D. Olafson, and W. A. Goddard, J. Phys. Chem. 94, 8897 (1990).

${ }^{29}$ W. L. Jorgensen, D. S. Maxwell, and J. Tirado-Rives, J. Am. Chem. Soc. 118 (45), 11225 (1996).

${ }^{30}$ N. P. Adhikari, X. H. Peng, A. Alizadeh, S. Ganti, S. K. Nayak, and S. K. Kumar, Phys. Rev. Lett. 93, 188301 (2004).

${ }^{31}$ C. Schliehe, B. H. Juarez, M. Pelletier, S. Jander, D. Greshnykh, M. Nagel, A. Meyer, S. Foerster, A. Kornowski, C. Klinke, and H. Weller, Science 329, 550 (2010).

${ }^{32}$ M. A. Sliem, A. Chemseddine, U. Bloeck, and R. A. Fischer, Cryst. Eng. Comm. 13, 483 (2011).

${ }^{33}$ I. Moreels, B. Frintzinger, J. C. Martins, and Z. Hens, J. Am. Chem. Soc. 130, 15081 (2008).

${ }^{34}$ G. Schaftenaar and J. Noordik, J. Comput.-Aided Mol. Des. 14, 123 (2000).

${ }^{35} \mathrm{~J}$. W. Ponder, tinker - software tools for molecular design, (2010); see http://dasher.wustl.edu/tinker/.

${ }^{36}$ N. Allinger, Y. Yuh, and J.-H. Lii, J. Am. Chem. Soc. 111, 8551 (1989)

${ }^{37}$ A. D. MacKerell, Jr., D. Bashford, M. Bellott, R. L. Dunbrack, Jr., J. D. Evanseck, M. J. Field, S. Fischer, J. Gao, H. Guo, S. Ha, D. Joseph-McCarthy, L. Kuchnir, K. Kuczera, F. T. K. Lau, C. Mattos, S. Michnick, T. Ngo, D. T. Nguyen, B. Prodhom, W. E. Reiher III, 
B. Roux, M. Schlenkrich, J. C. Smith, R. Stote, J. Straub, M. Watanabe, J. Wiorkiewicz-Kuczera, D. Yin, and M. Karplus, J. Phys. Chem. B 102(18), 3586 (1998).

${ }^{38}$ K. H. Chen, J. H. Lii, G. A. Walker, Y. Xie, H. F. Schaefer III, and N. L. Allinger, J. Phys. Chem. A 110, 7202 (2006).

${ }^{39}$ K. H. Chen, G. A. Walker, and N. L. Allinger, J. Mol. Struct.: THEOCHEM 490, 87 (1999).

${ }^{40}$ J. E. Goose and P. Clancy, J. Phys. Chem. C 111, 43 (2007).

${ }^{41}$ J. E. Goose, E. L. First, and P. Clancy, Phys. Rev. B 81, 205310 (2010).

${ }^{42}$ R. Cantrell and P. Clancy, Surf. Sci. 602(22), 3499 (2008).

${ }^{43}$ A. P. Kaushik and P. Clancy, Surf. Sci. 605(13-14), 1185 (2011).

${ }^{44}$ J. Nocedal, Math. Comput. 35, 773 (1980).

${ }^{45}$ J. J. Choi, C. R. Bealing, K. Bian, K. J. Hughes, W. Zhang, D. M. Smilgies, R. G. Hennig, J. R. Engstrom, and T. Hanrath, J. Am. Chem. Soc. 133(9), 3131 (2011)

${ }^{46}$ G. J. Martyna, D. J. Tobias, and M. L. Klein, J. Chem. Phys. 101, 4177 (1994).

${ }^{47}$ M. Parrinello and A. Rahman, J. Appl. Phys. 52, 7182 (1981).

${ }^{48}$ W. Shinoda, M. Shiga, and M. Mikami, Phys. Rev. B 69, 134103 (2004).

${ }^{49}$ M. E. Tuckerman, J. Alejandre, R. Lopez-Rendon, A. L. Jochim, and G. J. Martyna, J. Phys. A 39, 5629 (2006)
${ }^{50}$ G. Ciccotti, M. Ferrario, J. T. Hynesa, and R. Kapral, Chem. Phys. 129, 241 (1989).

${ }^{51}$ E. Guardia, R. Rey, and J. A. Padro, Chem. Phys. 155, 187 (1991).

${ }^{52}$ C. Jarzynski, Phys. Rev. Lett. 78, 2690 (1997).

${ }^{53}$ C. Jarzynski, Phys. Rev. E 56, 5018 (1997).

${ }^{54}$ S. J. Plimpton, J. Comput. Phys. 117, 1 (1995), see http://lammps.sandia.gov.

${ }^{55}$ M. P. Allen and D. J. Tildesley, Computer Simulation of Liquids (Clarendon, Oxford, 1987).

${ }^{56}$ P. Kr. Ghorai and S. C. Glotzer, J. Phys. Chem. C 111, 15857 (2007).

${ }^{57} \mathrm{~T}$. Hanrath, private communication (2011); see details in the supplementary material (Ref. 59).

${ }^{58}$ Y. Li, S.-T. Lin, and W. A. Goddard III, J. Am. Chem. Soc. 126, 1872 (2004).

${ }^{59}$ See supplementary material at http://dx.doi.org/10.1063/1.3689973 for some figures of isolated nanocrystals with ligands grafted on at different grafting densities as well as some plots of the variation of interaction energy between two nanocrystals as a function of ligand grafting density and ligand length. The supplemenary material provides some experimental data from differential scanning calorimetry to investigate ligand "bundling". The tables containing the force field parameters for the OPLS UA model (Ref. 19) as well as the Paul et al. UA model (Ref. 26) are also provided. 